\title{
An Optimized Algorithm for the Evaluation of Local Singularity Exponents in Digital Signals
}

\author{
Oriol Pont ${ }^{1, \star}$, Antonio Turiel ${ }^{2}$, and Hussein Yahia ${ }^{1}$ \\ 1 INRIA Bordeaux Sud-Ouest \\ 351 Cours de la Libération bt. A29bis, 33405 Talence \\ 2 Institut de Ciències del Mar, ICM - CSIC
}

Passeig marítim de la Barceloneta 37-49, 08003 Barcelona, Catalonia

oriol.pont@inria.fr

\begin{abstract}
Recent works show that the determination of singularity exponents in images can be useful to assess their information content, and in some cases they can cast additional information about underlying physical processes. However, the concept of singularity exponent is associated to differential calculus and thus cannot be easily translated to a digital context, even using wavelets. In this work we show that a recently patented algorithm allows obtaining precise, meaningful values of singularity exponents at every point in the image by the use of a discretized combinatorial mask, which is an extension of a particular wavelet basis. This mask is defined under the hypothesis that singularity exponents are a measure not only of the degree of regularity of the image, but also of the reconstructibility of a signal from their points.
\end{abstract}

\section{Introduction}

Since the introduction of wavelet theory, it has been recognized that the calculation of local singularity exponents from digital signals can be used to codify them in a more compact way [1019. The early studies carried out over turbulent flows and other systems proved that the singularity exponents at the top points in a Wavelet Transform Modulus Maxima (WTMM) line can be easily calculated 11|12|13. However, the extension of this methodology to any point at resolution scale was far from simple, and for those points the WTMM method become convoluted and rather imprecise [20, even for the mere assessment of the statistical properties of the signal 29].

A different approach using numerical determinations of the local gradient modulus convolved with wavelets, even with positive multiscaling bases, showed more stable results over discretized signals [25]27, leading to accurate exponents values and fine spatial resolution 21. This new approach to singularity analysis is in the basis of the so-called Microcanonical Multiscale Formalism (MMF) [32, which has been shown to be useful to assess physical properties of turbulent flows and other multiscale systems [24|6|31|32|28|15|17]16]. It has been hence

\footnotetext{
^ Corresponding author.
} 
demonstrated that obtaining the singularity exponents at each point of a signal can reveal many useful information not only about the image, but also about the physical processes giving raise to it.

However, the performance of MMF-based singularity analysis (namely, the quality of the exponents calculated with this approach) depends on the properties of the multiscaling function used [32, the best multiscaling functions being positive functions with fast enough decay [21. Although for statistical analysis almost any scaling function gives the same results [29, a precise geometrical determination of the underlying patterns, edges and textures requires a very fine tuning of the multiscaling function.

In this paper we discuss a method for singularity analysis introduced in a recent patent 22. This method allows high-performance determination of the singularity exponents, with a method which is fast, computationally cheap, stable, accurate and provides fine resolution. Its definition relies in the connection of singularity exponents with the concept of image reconstruction from the Most Singular Component (MSC), as presented in [30]. The paper is structured as follows: in the next Section we introduce the basics of singularity analysis, while Section 3 explains the key concept of reconstruction from the MSC. In Section 4 the conditions to define a UPM-based measure are discussed, while Section 5 gives the settings for the calculus on reduced neighborhoods. Finally, in Section 6 our method is presented and some results shown. The last Section, Section 7. presents the conclusions of our work.

\section{Definition of Singularity Analysis}

Singularity analysis is a term referring to different meanings in mathematical analysis (e.g. the studies of singularities of differentiable functions); in the present work we focus on its meaning in the theory of complex systems. Using singularity analysis we intend to describe and characterize the local behavior of a $\mathbb{R}^{m}$-valued function $f(\mathbf{x})$ defined on $\mathbb{R}^{d}$ around each one of its domain points $\mathbf{x}$ according to the so-called singularity exponent, Hölder exponent 7 or Hurst exponent 1918 . If the signal behaves at point $\mathrm{x}$ according to the following limiting behavior:

$$
\|f(\mathbf{x}+\mathbf{r})-f(\mathbf{x})\|=\alpha(\mathbf{x}) r^{h(\mathbf{x})}+o\left(r^{h(\mathbf{x})}\right) \quad(\mathbf{r} \rightarrow 0)
$$

then $h(\mathbf{x})$ is the singularity exponent at $\mathbf{x}$ : small displacements around $\mathbf{x}$ lead to function increment which scale as powers of the displacement modulus $r=$ $\|\mathbf{r}\|$. A strictly $n$-times derivable function obviously leads to a Hölder exponent $h(\mathbf{x})=n$, and so this formulation allows to generalize the concept of integer differentiability to real differentiability. To complete the transposition, a slightly more exigent formulation of eq. (1) is required, namely we should assume that there exists a $(1,1)$ continuous tensor from $\mathbb{R}^{d}$ to $\mathbb{R}^{m}, \alpha(\mathbf{x})$, such that

$$
f(\mathbf{x}+\mathbf{r})-f(\mathbf{x})=\langle\alpha(\mathbf{x}) \mid \mathbf{r}\rangle r^{h(x)-1}+o\left(r^{h(\mathbf{x})}\right) \quad(\mathbf{r} \rightarrow 0)
$$


(denoting $\langle\alpha(\mathbf{x}) \mid \mathbf{r}\rangle$ the standard duality bracket for $(1,1)$ tensors). When such representation is possible, the exponent $h(\mathbf{x})$ is called the Hurst exponent of the function; if only eq. (11) can be applied we will prefer to speak about Hölder exponents.

Assessment based on Hölder exponents can only be applied to very specific signals; in general, the presence of long range correlations and the effects of noise and discretization would preclude a direct evaluation of the scaling exponent [27/32].

A more general framework is given by singularity exponents, which are defined using gradient-based measures [27. Given a signal $s(\mathbf{x})$, defined in $\mathbb{R}^{d}$ and with values in $\mathbb{R}$, we can define its associated gradient measure $\mu$ by its density $\mathrm{d} \mu(\mathbf{x})$, which is given by:

$$
\mathrm{d} \mu(\mathbf{x})=\|\nabla s\|(\mathbf{x}) \mathrm{d} \mathbf{x}
$$

This measure is by definition absolutely continuous with respect to Lebesgue measure. Hence, the measure of any Borelian $\mathcal{A}$ is given by:

$$
\mu(\mathcal{A})=\int_{\mathcal{A}} \mathrm{d} \mathbf{x}\|\nabla s\|(\mathbf{x})
$$

Gradient measures also allow to characterize the local singularity of any point, and in a direction-independent manner. Following eq. (1), let us consider a function $f(\mathbf{x})$ with a Hölder exponent $h(\mathbf{x})+1$ at a point $\mathbf{x}$ (notice the shift +1 introduced for later convenience). Let $\mathcal{B}_{r}(\mathbf{x})$ be the ball (using an arbitrary norm in $\mathbb{R}^{d}$ ) of radius $r$ centered around $\mathbf{x}$. So, we obtain [27]:

$$
\mu\left(\mathcal{B}_{r}(\mathbf{x})\right)=\alpha(\mathbf{x}) r^{d+h(\mathbf{x})}+o\left(r^{d+h(\mathbf{x})}\right)(r \rightarrow 0)
$$

where $d$ is the dimension of the domain space ( $d=2$ in images). The introduction of gradient measures is convenient, as measures can also be wavelet-projected to obtain smooth interpolations from discretized data. Given a wavelet $\Psi$, we define 3112 the wavelet projection of the measure $\mu$ at the point $\mathbf{x}$ and scale $r$, denoted by $\mathcal{T}_{\Psi} \mu(\mathbf{x}, r)$, as:

$$
\mathcal{T}_{\Psi} \mu(\mathbf{x}, r)=\int_{\mathbb{R}^{d}} \mathrm{~d} \mu\left(\mathbf{x}^{\prime}\right) \frac{1}{r^{d}} \Psi\left(\frac{\mathbf{x}-\mathbf{x}^{\prime}}{r}\right)
$$

i.e. operator $\mathcal{T}_{\Psi}$ is a map from the set $\mathcal{M}$ of $\sigma$-finite measures on $\mathbb{R}^{d}$ to the set of functions $\mathbb{R}^{d} \times \mathbb{R}^{+} \rightarrow \mathbb{R}$. If the signal possesses a singularity exponent at the point $\mathbf{x}$ according to eq. (5), then the wavelet projections allow to infer this same exponent, as they verify [3]27]:

$$
\mathcal{T}_{\Psi} \mu(\mathbf{x}, r)=\alpha_{\Psi}(\mathbf{x}) r^{h(\mathbf{x})}+o\left(r^{h(\mathbf{x})}\right)(r \rightarrow 0)
$$

The main advantage of using measures over discretized data is that any function $\Psi$ can be used to evaluate singularity exponents using eq. (77), even positive functions 32. As discussed in [29, the resolution capability of a wavelet depends on the number of zero-crossings it has, which is increased in higher-order 
wavelets but is minimum for positive wavelets. So, gradient measures improve the spatial resolution of singularity exponents [21].

\section{$3 \quad$ MSC and Its Connection with Reconstruction}

An important ingredient in the construction of wavelets with optimized resolution capability is the concept of reconstruction of signals from partial information about its gradient. The theoretical and practical implementation of this reconstruction algorithm was first introduced in [30] (see discussion there).

We consider signals having a singularity exponent $h(\mathbf{x})$ at each point [14/5] and for which these exponents are organized forming sets with a particular multiscale structure [4. That is, the values of the singularity exponents do not take arbitrary values but must be organized so that they define a hierarchy of multiscale geometrical structures "matching" and realizing closely the cascading properties of some random variables associated to the macroscopic description of the system under study [18. Due to the difficulties of classical methodologies to assign a precise value of singularity exponent $h(\mathbf{x})$ to each point, all the characterizations of this hierarchy that have been tried up to now are merely statistical. In 30] a new question was posed: if the hierarchy truly exists in complex signals, can they be reconstructed starting from the vertex of this hierarchy? For multifractals, the set associated to the vertex is well-known, at least from the theoretical point of view: it is the so-called Most Singular Component (MSC), which is the set comprising the points with most singular (i.e., most negative) values of $h(\mathbf{x})$ [27/32].

The thesis in [30] is that the MSC contains enough information to fully reconstruct the signal (in that reference, the reconstruction of images is analyzed, although the formulas are valid for any number of dimensions). As we are working with gradient measures, the data to be retained at the MSC is the gradient of the signal. So, it was hypothesized that there exists an universal operator to reconstruct signals starting from the values of the signal, and leading to a reconstruction algorithm consistent with the known statistical invariances of turbulence and multiscale signals 5 . The algorithm was required to be deterministic, linear, translational invariant, isotropic and leading to the known power-spectrum shape. Under these requirements, it turned out that there exists, if any, only one possible operator to reconstruct signals from the gradient on the MSC. Let us first define a convenient notation for the starting data. For a given multiscale signal $s$ let us denote by $\mathcal{F}_{\infty}$ the MSC, that is to say $\mathcal{F}_{\infty}$ is the set of points $\mathbf{x}$ such that $h(\mathbf{x}) \in] h_{\infty}-\Delta, h_{\infty}+\Delta\left[\right.$ with $h_{\infty}$ being the minimum value of all $h(\mathbf{x})$ over the finite domain of the discrete signal, and $\Delta$ a threshold parameter; we will define the essential gradient of $s, \nabla_{\mathcal{F}_{\infty}} s$, as follows:

$$
\nabla_{\mathcal{F}_{\infty}} s(\mathbf{x})=\nabla s(\mathbf{x}) \delta_{\mathcal{F}_{\infty}}(\mathbf{x})
$$

where $\delta_{\mathcal{F}_{\infty}}$ is a delta distribution associated to the continuum of the $\mathcal{F}_{\infty}$, homogeneous in (Hausdorff) topological dimension to a repartition in between 
dimensions $d-1$ and $d$ : it assigns uniform weight to the points on the $\mathrm{MSC} \mathcal{F}_{\infty}$ and vanishes outside the MSC. According to this notation, the reconstruction formula 30] reads:

$$
s(\mathbf{x})=\left(\boldsymbol{g} \cdot \nabla_{\mathcal{F}} s\right)(\mathbf{x})
$$

where the symbol - means convolution dot-product of vectors and the vector field $\boldsymbol{g}$ is the universal reconstruction kernel, which can be easily expressed in Fourier space, namely:

$$
\hat{\boldsymbol{g}}(\mathbf{k})=i \frac{\mathbf{k}}{\|\mathbf{k}\|^{2}}
$$

and $i=\sqrt{-1}$ is the imaginary unit. So defined, the reconstruction kernel $\boldsymbol{g}$ is a kind of inverse gradient operator. There is always a set $\mathcal{F}_{\infty}$ from which reconstruction is perfect, the whole domain: If $\mathcal{F}_{\infty}=\mathbb{R}^{d}$, then eq. (9) reduces to the trivial identity $\nabla_{\mathcal{F}} s=\nabla s$. But from eq. (9) is not evident if there exists a smaller set $\mathcal{F}_{\infty} \subset \mathbb{R}^{d}$ such that reconstruction is also perfect. Following the derivation in [30], we can conclude that any set $\mathcal{F}$ leading to a perfect reconstruction must verify:

$$
\operatorname{div}\left(\nabla_{\mathcal{F} c} s\right)=0
$$

where $\mathcal{F}^{c}$ is the complementary set of $\mathcal{F}$. As the divergence operator is local and the formula above is linear, the decision to include or not a point can be taken on the basis of any neighborhood around that point. The points that must always be included to obtain a perfect reconstruction are hence those with values that cannot be predicted just knowing the values in their surroundings; they are hence called unpredictable points (in opposition to the other points, which are predictable). Predictability is a subject at the core of the analysis of complex systems and signals [21], where for instance Lyapunov exponents and Kolmogorov-Sinai entropy are known measures of information growth in a dynamical system. The set $\mathcal{F}_{u}$ formed by the collection of all the unpredictable points is what we will call the Unpredictable Points Manifold (UPM) and it is, by definition, the smallest set for which eq. (9) lead to a perfect reconstruction. The hypothesis in [30] is that $\mathcal{F}_{u}=\mathcal{F}_{\infty}$, a conjecture which is at the base of the framework of reconstructible systems. What is evident from experiences is that the MSC leads to good reconstructions (see discussion in [32]).

\section{General Conditions to Define UPM-Measures}

We now step forward to generalize the concept of gradient measure introduced in the previous sections to the novel concept of UPM-measure. The basic requirements to define a singular positive UPM-measure $\mu$ are:

i) It is concerned with the local singular behavior of functions.

ii) It leads to a MSC as close to the UPM as possible. 
In some sense, UPM-measures are gradient measures which also take into account the degree of predictability of points according to eq. (11). So that, they take the form of a gradient (in the sense of finite difference over discretized signals) but with penalty terms associated to the lack of predictability. The best way to keep on working around singularities is to define UPM-measures as vectorial wavelet projections of standard gradient measures. So, the UPM-measure is a carefully designed vectorial wavelet projection of the gradient measure so that it penalizes unpredictability.

In our method, in contrast with standard singularity analysis, we will not perform many wavelet projections of the UPM measure in order to extract the singularity exponents by means of a log-log regression applied to eq. (77). Waveletprojecting the measure at several scales is costly in computer time and only serves to enhance the resolution of less singular structures at the cost of coarsening most singular ones (see a discussion on this in [29]). But as we are mainly interested in the most singular structures, it is hence harmful to our interests to project across multiple scales. Instead, we will make use of point estimates [29]15] of the singularity exponents, namely:

$$
h(\mathbf{x})=\frac{\log \left(\mathcal{T}_{\Psi} \mu\left(\mathbf{x}, r_{0}\right) /\left\langle\mathcal{T}_{\Psi} \mu\left(\cdot, r_{0}\right)\right\rangle\right)}{\log r_{0}}+o\left(\frac{1}{\log r_{0}}\right)
$$

where $\left\langle\mathcal{T}_{\Psi} \mu\left(\cdot, r_{0}\right)\right\rangle$ is the average value of the wavelet projection over the whole signal and serves to diminish the relative amplitude of the $o\left(\frac{1}{\log r_{0}}\right)$ correction. When applying eq. (12) we will need that $r_{0}$ is small enough to neglect this correction. The scale $r_{0}$ will be defined as the smallest accessible one, that is, the pixel scale. We conventionally assign a Lebesgue measure of 1 to the whole space domain, so for a $N \times M$ image the value of $r_{0}$ is fixed to $r_{0}=\frac{1}{\sqrt{N M}}$, so in general we need that images are large enough to make the first term in the right hand side of eq. (12) a good approximation of the singularity exponent. In practical terms, this implies a resolution around $100 \times 100$ pixels or larger.

\section{Calculus on Reduced Neighborhoods: Cross Fourier Transform}

In order to assess the degree of predictability of a given point, we will apply the reconstruction formula, eq. (9), for the smallest possible neighbor of a point, namely its $2 d$ nearest neighbors in $2 d$ connexity neighborhoods. In $2 \mathrm{D}(d=2)$ this consists of 4 neighbors, that with the point altogether form a cross. For any quantity $p(\mathbf{x})$ we will represent the neighborhood of any point $\mathbf{x}_{0}$ by a 5 component vector comprising this point and its 4 nearest neighbors, following the indexing convention established in Figure 1. So, the central point will be assigned the index 0 , the point at its right will be indexed 1 , the one on the left is indexed 2, that on top is indexed as 3 and the one on the bottom is indexed 4 . So, we convert the neighbor in the vector $\left(p_{0}, p_{1}, p_{2}, p_{3}, p_{4}\right)$. The notion of predictable point easily extends to any number of dimensions, regardless of the 


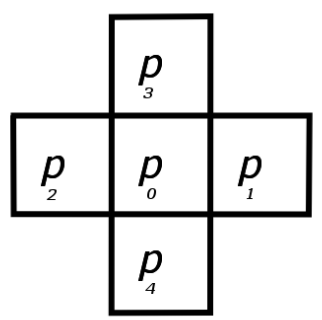

Fig. 1. Schematic representation of the indexing of the points in the $2 \mathrm{D}$ cross

number of components of the neighbor vector, which grows as the dimension $d$ increases.

We could apply the harmonics of the standard Fourier Transform on the discrete signal $\left(p_{0}, p_{1}, p_{2}, p_{3}, p_{4}\right)$, but this is not a good idea. The harmonics of the standard Fourier Transform (i.e., $\left.\left(e^{2 i k \pi / n}\right)_{k}\right)$ depend on the size of the embedding space, so they would lead to a dimension-dependent measure of the predictability . To overcome this problem, we note that relative to the center of the cross, the position of the other points correspond to displacements of \pm 1 (in pixel units) either in the $x$-direction or in the $y$-direction. So that, to define a special type of Fourier transform specialized to this cross formation, the basic Nyquist frequency in each direction is $2 \pi / 3$. Consequently we introduce

$$
j=e^{2 \pi i / 3}=\cos (2 \pi / 3)+i \sin (2 \pi / 3)=-\frac{1}{2}+i \frac{\sqrt{3}}{2}, \bar{j}=j^{2}
$$

We define the direct Cross Fourier Transform of any 5 -vector $\boldsymbol{p}=\left(p_{0}, p_{1}, p_{2}, p_{3}, p_{4}\right)$ as the complex 5 -vector $\hat{\boldsymbol{p}}=\left(\hat{p}_{0}, \hat{p}_{1}, \hat{p}_{2}, \hat{p}_{3}, \hat{p}_{4}\right)$ obtained according to the following formula:

$$
\hat{\boldsymbol{p}}=\mathbb{F} \boldsymbol{p}
$$

where $\mathbb{F}$ is the following $5 \times 5$ complex matrix:

$$
\mathbb{F}=\frac{1}{3}\left[\begin{array}{lllll}
1 & 1 & 1 & 1 & 1 \\
1 & j & j & 1 & 1 \\
1 & j & j & 1 & 1 \\
1 & 1 & 1 & j & j \\
1 & 1 & 1 & j & j
\end{array}\right]
$$

This matrix represents the linear combination of the harmonics associated to the displacements in the cross and is designed to represent with the maximum fidelity the composition at the center of the cross, starting from the nearest points. The inverse of this matrix is: 


$$
\mathbb{F}^{-1}=\left[\begin{array}{ccccc}
-1 & 1 & 1 & 1 & 1 \\
1 & j & j & 0 & 0 \\
1 & j & \bar{j} & 0 & 0 \\
1 & 0 & 0 & \bar{j} & j \\
1 & 0 & 0 & j & \bar{j}
\end{array}\right]
$$

We need to define surrogates of the gradient and the reconstruction formula restricted to the cross neighborhood, in order to evaluate in a fast way the degree of predictability of the central point. For that reason, we will construct appropriate implementations of the gradient and of the gradient reconstruction formula, based on the Cross Fourier Transform.

The Cross Gradient Operator is the operator $\left(\partial_{x}, \partial_{y}\right)=\mathbb{F}^{-1} \cdot\left(\hat{\partial_{x}}, \hat{\partial_{y}}\right) \cdot \mathbb{F}$. In Fourier space the operator acts by simply multiplying any function by the functions $\hat{\partial}_{x}$ and $\hat{\partial_{y}}$ to obtain the $x$ and the $y$ coordinate, respectively. The function $\hat{\partial_{x}}$ is defined as:

$$
\hat{\partial_{x}}=(0, i \sqrt{3},-i \sqrt{3}, 0,0)
$$

and analogously we have:

$$
\hat{\partial_{y}}=(0,0,0, i \sqrt{3},-i \sqrt{3})
$$

The Cross Reconstruction Operator is one of the inverses of the Cross Gradient Operator. As the gradient operator eliminates any constant summed up to each component of the 5-vector representing the neighborhood, the reconstruction is defined up to a constant shift; our implementation of the cross reconstruction operator is such that the 5 -vector has zero mean, $\sum_{i=1}^{5} s_{i}=0$. For that reason, signals should have the mean subtracted before applying these two operators (see below).

The Cross Reconstruction is the operator $R=\mathbb{F}^{-1} \cdot \hat{R} \cdot \mathbb{F}$. In Fourier space $\hat{R}$ has two functional components, $\hat{R}=\left(\hat{R}_{x}, \hat{R}_{y}\right)$; the operator acts as the sum of the product of each component with the corresponding component (x and y) of the gradient on which it is operated. The component $\hat{R}_{x}$ is defined as:

$$
\hat{R}_{x}=(0,-i / \sqrt{3}, i / \sqrt{3}, 0,0)
$$

and analogously for $\hat{R}_{y}$,

$$
\hat{R}_{y}=(0,0,0,-i / \sqrt{3}, i / \sqrt{3})
$$

The Cross Gradient and the Cross Reconstruction are the two basic algorithms for the design of the UPM-measures. They can be simplified to a $5 \times 5$ matricial form, for faster numeric implementation.

\section{Local Correlation Singularity Measure}

The Local Correlation Singularity Measure is designed to measure the unpredictability of a given point, just quantifying the difference on the actual 
value of the detrended (i.e., after subtracting the mean) signal at a given point and the inferred one from their four neighbors. It is defined algorithmically as follows:

Goal: To evaluate $\mathcal{T}_{\Psi_{l c s m}} \mu\left(\mathbf{x}_{0}, r_{0}\right)$ at a given point $\mathbf{x}_{0}$.

\section{Algorithm}

1. The neighborhood of $\mathbf{x}_{0}$ is converted into a 5-vector $s=\left(s_{0}, s_{1}, s_{2}, s_{3}, s_{4}\right)$ according to the scheme in Figure 1

2. The vector is conveniently detrended: we first obtain $\bar{S}=\frac{1}{3} \sum_{i=1}^{5} s_{i}$, and we define the detrended vector, $\boldsymbol{p}=\left(p_{0}, p_{1}, p_{2}, p_{3}, p_{4}\right)$ as:

$$
p_{0}=s_{0}+\bar{S} ; p_{i}=s_{0}-\bar{S}, i=1, \ldots, 4
$$

3. We apply the Cross Gradient Operator to $\boldsymbol{p}$, so we obtain the vector $\boldsymbol{g}_{x}$ and $\boldsymbol{g}_{y}$.

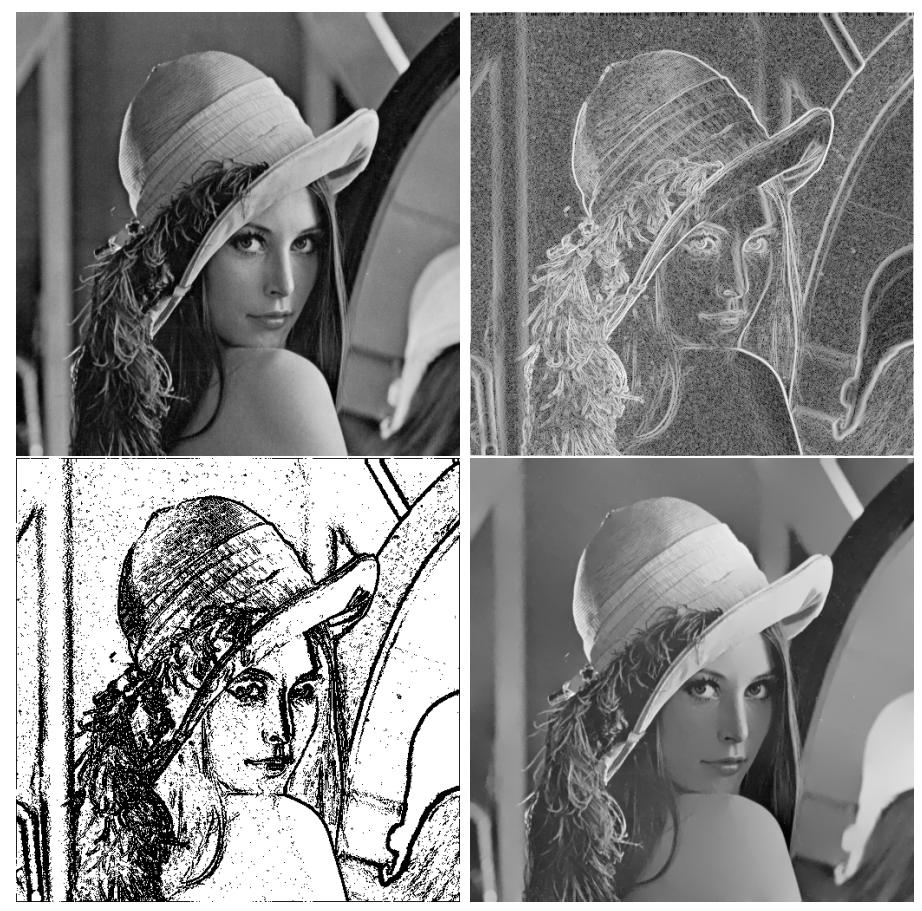

Fig. 2. Top: Left: Original Lena image; Right: Singularity exponents estimated using the Local Correlation Singularity Measure; they are represented using a inverse grayscale palette (the brightest the smaller, so more singular). Bottom: Left: MSC, defined as $\{h<-0.5\}$; it comprises $30 \%$ of the points of the image ; Right: Reconstruction. Some details are missing due to the lack of capability of the method to capture every UPM point; however, the reconstruction is of high quality $(24.5 \mathrm{~dB})$. 
4. We keep the value of the first components of these two vectors for a later use, $A_{x}=g_{x, 0}, A_{y}=g_{y, 0}$.

5. We set these two components to zero, $g_{x, 0}=g_{y, 0}=0$.

6. We apply the Cross Reconstruction Operator to the resulting vectors $\boldsymbol{g}_{x}$ and $\boldsymbol{g}_{y}$, to obtain the reconstructed signal $\boldsymbol{r}$.

7. We apply once more the Cross Gradient Operator onto $\boldsymbol{r}$ to obtain $\boldsymbol{\rho}_{x}$ and $\rho_{y}$.

8. We define the Local Correlation Singularity Measure as the modulus of the difference of the cross gradients at the center of the cross, namely:

$$
\mathcal{T}_{\Psi_{l c s m}} \mu\left(\mathbf{x}_{0}, r_{0}\right)=\sqrt{\left(A_{x}-\rho_{x, 0}\right)^{2}+\left(A_{y}-\rho_{y, 0}\right)^{2}}
$$

In fact, this last step means to keep the modulus of a vector-valued wavelet projection, but to simplify notation we leave it as is.

9. The singularity exponent $h\left(\mathbf{x}_{0}\right)$ is then obtained in application of eq. (12).

In Figure 2 we show an example of the application of the singularity analysis based on the Local Correlation Singularity Measure.

\section{Conclusions}

The accurate estimation of singularity exponents in multiscale systems allows characterizing their relevant features and identifying their information content. This is particularly important for the case of digital images, where the degree of singularity is directly related to the distribution of information, and so its knowledge can be used for compact coding or reconstructing from the Most Singular Component (MSC). However, digital images are discretized and this fact is an important obstacle for precisely retrieving its singularity exponents: even when using wavelet projections, most standard wavelet bases only give average results.

In this article, we have presented a recent algorithm that allows obtaining the singularity exponents of an image at every point. The singularity exponents are extracted in a precise and meaningful way, by means of a discretized combinatorial mask. This mask is constructed by considering the singularity exponent of a given point as both a measure of the singularity/regularity degree and a measure of the unpredictability of that point. The result is a discretized, numerical extension of a particular wavelet basis.

We have presented and discussed the method for singularity analysis noted as "Local Correlation Singularity Measure" in patent [22]. This method attains at the same time good quality and spatial resolution in the estimation of singularity exponents. Additionally, the reconstruction from the MSC is of high quality. As an illustration, we have shown the singularity exponents from the Local Correlation Singularity Measure and the reconstruction from their MSC for Lena's image, for which the reconstruction quality is of $24.5 \mathrm{~dB}$. The prospects of this method includes image compression [30, assessment of streamlines in turbulent 
flows [3126], detection of convection meteorological systems [23] or detection of investment cycles in stock market series [28, among others.

The presented methodology for $2 \mathrm{D}$ images can be easily generalized to any dimensionality. In addition, it is possible to define other UPM-measures other than the Local Correlation Singularity Measure, that can give better performance in certain cases. All these additional developments and their respective applications will be the object of future communications.

\section{References}

1. Aurell, E., Boffetta, G., Crisanti, A., Palading, G., Vulpiani, A.: Predictability in the large: an extension of the concept of lyapunov exponent. Journal of Physics A 30, 1-26 (1997)

2. Boffetta, G., Cencini, M., Falcioni, M., Vulpiani, A.: Predictability: a way to characterize complexity. Physics reports 356(6), 367-474 (2001)

3. Daubechies, I.: Ten lectures on wavelets. CBMS-NSF Series in App. Math. Capital City Press, Montpelier (1992)

4. Falconer, K.: Fractal Geometry: Mathematical Foundations and Applications. John Wiley and Sons, Chichester (1990)

5. Frisch, U.: Turbulence: The legacy of A.N. Kolmogorov. Cambridge Univ. Press, Cambridge (1995)

6. Isern-Fontanet, J., Turiel, A., Garcia-Ladona, E., Font, J.: Microcanonical multifractal formalism: application to the estimation of ocean surface velocities. Journal of Geophysical Research 112, C05024 (2007)

7. Jaffard, S.: Multifractal formalism for functions. I. Results valid for all functions. SIAM Journal of Mathematical Analysis 28(4), 944-970 (1997)

8. Jones, C.L., Lonergan, G.T., Mainwaring, D.E.: Wavelet packet computation of the hurst exponent. J. Phys. A: Math. Gen. 29(10), 2509 (1996)

9. Mallat, S., Huang, W.L.: Singularity detection and processing with wavelets. IEEE Trans. in Inf. Th. 38, 617-643 (1992)

10. Mallat, S., Zhong, S.: Wavelet transform maxima and multiscale edges. In: Ruskai, M.B., et al. (eds.) Wavelets and their Applications. Jones and Bartlett, Boston (1991)

11. Mallat, S., Zhong, S.: Characterization of signals from multiscale edges. IEEE Trans. on Pattern Analysis and Machine Intelligence 14, 710-732 (1992)

12. Mallat, S.: A Wavelet Tour of Signal Processing, 2nd edn. Academic Press, London (1999)

13. Muzy, J.F., Bacry, E., Arneodo, A.: Wavelets and multifractal formalism for singular signals: Application to turbulence data. Physical Review Letters 67, 3515-3518 (1991)

14. Parisi, G., Frisch, U.: On the singularity structure of fully developed turbulence. In: Ghil, M., Benzi, R., Parisi, G. (eds.) Turbulence and Predictability in Geophysical Fluid Dynamics. Proc. Intl. School of Physics E. Fermi, pp. 84-87. North Holland, Amsterdam (1985)

15. Pont, O., Turiel, A., Pérez-Vicente, C.: Application of the microcanonical multifractal formalism to monofractal systems. Physical Review E 74, 61110 (2006)

16. Pont, O., Turiel, A., Pérez-Vicente, C.: Description, modeling and forecasting of data with optimal wavelets. Journal of Economic Interaction and Coordination 4, 39-54 (2009) 
17. Pont, O., Turiel, A., Pérez-Vicente, C.: Empirical evidences of a common multifractal signature in economic, biological and physical systems. Physica A 388, 2025-2035 (2009)

18. She, Z.S., Leveque, E.: Universal scaling laws in fully developed turbulence. Physical Review Letters 72, 336-339 (1994)

19. Simonsen, I., Hansen, A., Magnar, O.: Determination of the hurst exponent by use of wavelet transforms. Phys. Rev. E 58(3), 2779-2787 (1998)

20. Struzik, Z.R.: Determining local singularity strengths and their spectra with the wavelet transform. Fractals 8(2), 163-179 (2000)

21. Turiel, A.: Relevance of multifractal textures in static images. Electronic Letters on Computer Vision and Image Analysis 1(1), 35-49 (2003)

22. Turiel, A.: Method and system for the singularity analysis of digital signals, patent registered under number PCT/ES2008/070195 (2008)

23. Turiel, A., Grazzini, J., Yahia, H.: Multiscale techniques for the detection of precipitation using thermal IR satellite images. IEEE Geoscience and Remote Sensing Letters 2(4), 447-450 (2005), doi:10.1109/LGRS.2005.852712

24. Turiel, A., Isern-Fontanet, J., García-Ladona, E., Font, J.: Multifractal method for the instantaneous evaluation of the stream function in geophysical flows. Physical Review Letters 95(10), 104502 (2005), doi:10.1103/PhysRevLett.95.104502

25. Turiel, A., Mato, G., Parga, N., Nadal, J.P.: The self-similarity properties of natural images resemble those of turbulent flows. Physical Review Letters 80, 1098-1101 (1998)

26. Turiel, A., Nieves, V., García-Ladona, E., Font, J., Rio, M.H., Larnicol, G.: The multifractal structure of satellite temperature images can be used to obtain global maps of ocean currents. Ocean Science 5, 447-460 (2009)

27. Turiel, A., Parga, N.: The multi-fractal structure of contrast changes in natural images: from sharp edges to textures. Neural Computation 12, 763-793 (2000)

28. Turiel, A., Pérez-Vicente, C.: Role of multifractal sources in the analysis of stock market time series. Physica A 355, 475-496 (2005)

29. Turiel, A., Pérez-Vicente, C., Grazzini, J.: Numerical methods for the estimation of multifractal singularity spectra on sampled data: a comparative study. Journal of Computational Physics 216(1), 362-390 (2006)

30. Turiel, A., del Pozo, A.: Reconstructing images from their most singular fractal manifold. IEEE Trans. on Im. Proc. 11, 345-350 (2002)

31. Turiel, A., Solé, J., Nieves, V., Ballabrera-Poy, J., García-Ladona, E.: Tracking oceanic currents by singularity analysis of micro-wave sea surface temperature images. Remote Sensing of Environment 112, 2246-2260 (2008)

32. Turiel, A., Yahia, H., Pérez-Vicente, C.: Microcanonical multifractal formalism: a geometrical approach to multifractal systems. Part I: Singularity analysis. Journal of Physics A 41, 15501 (2008) 